\title{
Cutaneous Myiasis of the Scalp Presenting as Bilateral Orbital Edema
}

\author{
Mandeep Kaur ${ }^{1}$, Inderjit Kaur ${ }^{2}$
}

\begin{abstract}
Background: Myiasis is the infestation of live human tissues by the larvae of flies, more commonly known as maggots. Wound myiasis occurs when fly larvae infest open wounds. This kind of infestation may be the result of facultative or obligatory parasites. Treatment with application of mineral oil and manual removal of maggots is the usual routine.

Case description: We report a case of 19-year-old female presenting with bilateral orbital edema. The differential diagnosis of eyelid erythema and edema is broad, ranging from benign, self-limiting dermatoses to malignant tumors and vision-threatening infections. History revealed that she had cutaneous myiasis of the scalp for which she was treated with mineral oil and maggots were removed manually a day prior to presentation. Irritant dermatitis by trickling of the mineral oil led to bilateral edema in this case. A definitive diagnosis usually can be made on physical examination of the eyelid, a careful evaluation of symptoms and history of exposure.

Conclusion: Detailed history and meticulous workup can be of great help in the differential diagnosis of bilateral orbital edema.

Keywords: Lesion, Myiasis, Orbital edema, Rare case, Risk factors, Scalp.

AMEl's Current Trends in Diagnosis \& Treatment (2020): 10.5005/jp-journals-10055-0099
\end{abstract}

\section{INTRODUCTION}

Myiasis as defined by Zumpt is the infestation of live human and vertebrate animals with dipterous larvae, which, at least for a certain period, feed on the host's dead or living tissue, liquid body substances or ingested food. ${ }^{1}$ Human myiasis is a rare clinical condition, but more frequently seen in tropical and subtropical areas. The first description of myiasis was given by Hope in $1840 .{ }^{2}$ The larvae have the ability to infect skin, dead tissues, and natural cavities of living persons. ${ }^{3}$ Ocular myiasis refers to infestations of the eye and/or ocular adnexa by larvae of the order Diptera and represents $<5 \%$ of the cases of human myiasis. ${ }^{4,5}$ It is divided into orbital, internal, or external, based on the site of larval infestation. Clinical manifestations may be varied depending upon the location of the larvae. External ocular myiasis refers to superficial infestation of ocular tissue. Periorbital edema is the proper term for puffy eyes causing swelling in the area around the eyes due to fluid buildup because of inflammation and gives the eye orbit a puffy appearance. It may be unilateral or bilateral, acute or chronic, and may cause from health to lifestyle - it may signal a serious underlying disease or may be temporary. It presents with mild to severe inflammation, diplopia, blurred vision, redness around eyes, bulging of eyes, conjunctival congestion, watering, photophobia, pain with or without movement of eyes, and itchiness around the eyes. We report a case of bilateral orbital edema as a rare presentation of cutaneous myiasis of the scalp.

\section{Case Description}

A 19-year-old female was referred from the surgery department of a tertiary care hospital and medical institute in Amritsar to the Eye OPD with one-day history of painless bilateral lid edema, itching, and inability to open her eyes. There was a history of fall from bed causing scalp injury few days back. The relatives showed her to local practitioner, and when she became a little disoriented, they brought her to this hospital. She belonged to a large Gujjar family

\begin{abstract}
1,2Department of Ophthalmology, Sri Guru Ram Das Institute of Medical Sciences and Research, Amritsar, Punjab, India

Corresponding Author: Mandeep Kaur, Department of Ophthalmology, Sri Guru Ram Das Institute of Medical Sciences and Research, Amritsar, Punjab, India, Phone: +91 8727088096, e-mail: sidhu_23@hotmail.co.in

How to cite this article: Kaur M, Kaur I. Cutaneous Myiasis of the Scalp Presenting as Bilateral Orbital Edema. AMEl's Curr Trends Diagn Treat 2020;4(2):107-109.
\end{abstract}

Source of support: Nil

Conflict of interest: None

residing in small kaccha house with poor hygienic conditions and low socioeconomic status dealing with cattle rearing. There was no travel history. On examination, her visual acuity was $20 / 20$, lids and forehead were edematous, mild erythema was seen, non-tender with slight mucoid discharge, conjunctiva showed superficial congestion and chemosis; however, the cornea was clear (Fig. 1). Further, it revealed that manual removal of maggots was done in the surgery ward and mineral oil was instilled on the wound for the same, an evening before being referred to the Eye OPD.

Examination of the scalp showed an open wound with loss of hair (Fig. 2); however, no live maggots were seen either at the wound site or in the eyes. There was no lymphadenopathy.

Laboratory investigations revealed hemoglobin $10 \mathrm{~g} / \mathrm{dl}$ and total leukocytes count $12 \times 10^{9} / \mathrm{L}$ with raised neutrophils. ESR $10 \mathrm{~mm} / \mathrm{hr}$, random blood sugar $140 \mathrm{mg} / \mathrm{dl}$, and viral markers were normal. X-ray skull did not show any abnormality.

\section{Treatment}

1. Dressing with paraffin followed by povidone iodine was done. The patient was treated with systemic antibiotics and analgesics. 


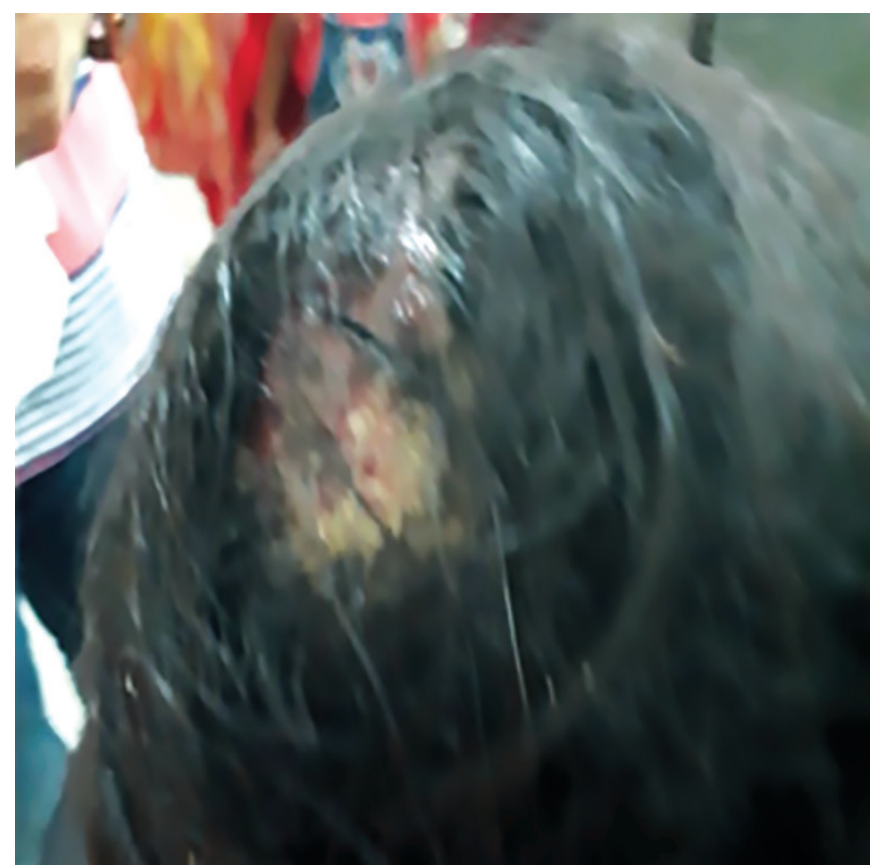

Fig. 1: Scalp wound

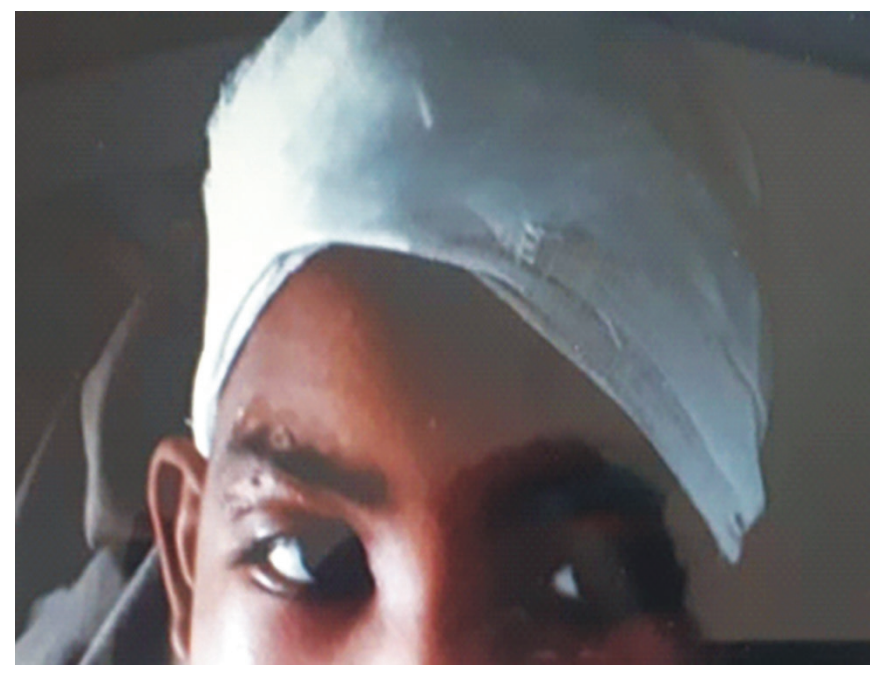

Fig. 2: At the time of discharge

2. For orbital edema, cold compresses were advised with oral anti-histaminics, topical corticosteroids, antibiotic eye drops 4 times/day, and lubricants thrice/day as prescribed.

3. Follow-up after 5 days showed complete resolution of orbital edema (Fig. 3)

\section{Discussion}

Although maggots or intact larvae were not seen on presentation to the ophthalmology clinic, the clinical history and features suggest bilateral contact dermatitis, which could be due to irritant mineral oil or larval secretions. Myiasis is caused by the invasion of tissues or organs of humans or animals by dipterous larvae. Human myiasis

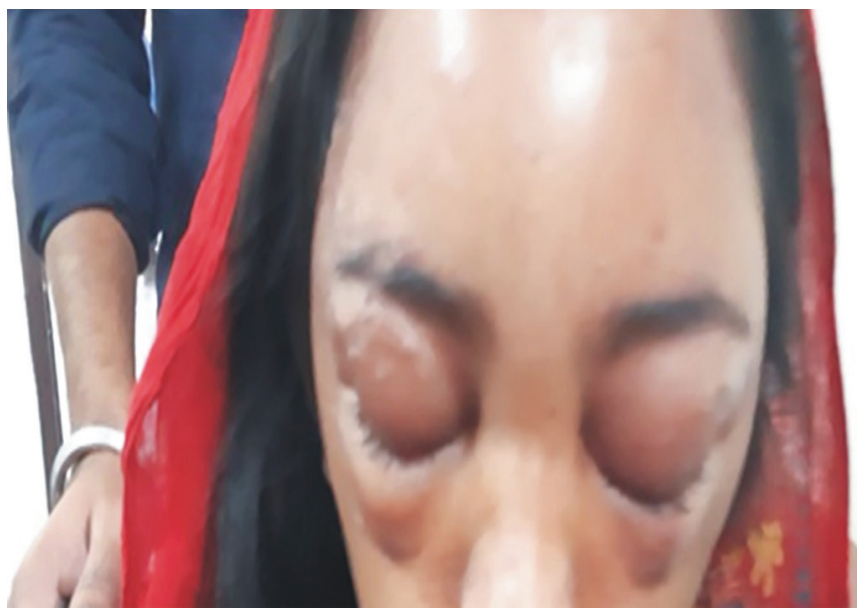

Fig. 3: At the time of presentation

Table 1: Classification of myiasis given by Bishopp's, James', and Zumpt's ${ }^{8}$

1. Sanguinivorous or bloodsucking

2. Wound myiasis

3. Cutaneous myiasis, furuncular, and migratory

4. Cavitary myiasis, where the infestation receives the name of the affected organ, e.g., cerebral myiasis, aural myiasis, nasal myiasis, and ophthalmomyiasis

is a rare clinical condition, but more frequently seen in tropical and subtropical areas. Hot, humid climate with inadequate sanitary conditions favors the development of this condition. Dermatitis, psychiatric illnesses, leprosy, and diabetes are some contributory factors. ${ }^{6}$ It is the fourth most common travel-associated skin disease, which can be divided according to the body's tissue vulnerability. The most common and the most frequently encountered clinical form is cutaneous myiasis, while body cavity, nasopharyngeal, ocular, aural, gastrointestinal, and urogenital myiases are less common ${ }^{7}$ as mentioned in Table 1.

Wound myiasis occurs when fly larvae infest open wounds, which may be the result of facultative or obligatory parasites. The larvae of the housefly Malus domestica are found in dung and decaying refuse and are saprophagous. Most Sarcophagidae species that cause myiasis deposit their eggs or larvae over the host at some predisposing site, such as wounding, necrosis, or bacterial contamination. ${ }^{9}$ Advanced age, poor social conditions, poor personal hygiene, poor general condition, mental retardation, immobilization, diabetes mellitus, alcoholism, vascular occlusive disease, ulcerating lesions, bacterial infection of wounds and infected dermatitis, travel to endemic areas, and contact with livestock are predisposing factors for cutaneous myiasis. ${ }^{7,10}$ Our case was also having wound myiasis, poor socioeconomic condition, poor personal hygiene, and contact with livestock though no similar case of distant cutaneous lesion of myiasis causing periorbital edema has been reported in the literature.

Contact dermatitis is the most common cause of cutaneous eyelid inflammation. ${ }^{11}$ The skin over the eyelid is vulnerable to irritants and allergens because of its thinness, sensitivity, and frequent exposure to chemicals via direct application or contamination from fingers and hands ${ }^{11,12}$ and hence may be the initial or the only presenting area with signs of contact dermatitis, 
while other areas of the body remain unaffected by the same exposure. ${ }^{12}$ Contact dermatitis can be classified as allergic or irritant. The presenting features of both types are often not readily distinguishable, but patients with irritant contact dermatitis often present with greater burning and stinging compared with the characteristic pruritus of allergic contact dermatitis ${ }^{12,13}$ as was present in our case. Contact dermatitis of the eyelid is mediated by a type IV hypersensitivity reaction in allergic contact dermatitis and by direct toxic effect in irritant contact dermatitis. ${ }^{13}$ It is more often caused by a product applied to the hair, nails, or face than by the products applied directly to the eyelids. ${ }^{12}$ Thus, in our case, the patient might have touched her lids with infested nails or hands and resulted in periorbital edema.

Various differentials for the swollen red eyelid include orbital cellulitis, which is a severe infection presenting with proptosis and ophthalmoplegia and life-threatening. Less serious conditions, such as contact dermatitis, atopic dermatitis, and blepharitis, are more common causes of eyelid erythema and edema and are differentiated on the basis of clinical clues such as time course, presence or absence of irritative symptoms, scaling, and other skin findings. Discrete lid lesions like vesicles, erosions, or crusting are also important diagnostic indicators for herpes infection. Hordeola, chalazia, and furuncles are benign, self-limiting eyelid nodules, whereas malignancies require surgical excision.

\section{Conclusion}

In human cutaneous myiasis, the lesions are often misdiagnosed as furunculosis, staphylococcal boil, insect bite, sebaceous cyst, cellulitis, and conditions with similar presentations. Thus, a correct diagnosis will help alleviate the anxiety of the patient and prevent the complications. Prevention and control of myiasis should include strategic planning that recommends basic cleaning and sanitary education programs for the community. Medical professionals should keep this diagnosis in their differential when examining patient with infected wound and orbital edema.

\section{References}

1. Zumpt F. Myiasis in Man and Animals in the Old World. London, UK: Butterworth \& Co.; 1965. pp. 1-107.

2. Hope FW. On insects and their larvae occasionally found in the human body. Trans R Soc Entomol 1840;2:256-271.

3. Francesconi F, Lupi O. Myiasis. Clin Microbiol Rev 2012;25:79-105. DOI: 10.1128/CMR.00010-11.

4. Gupta PC, Ram J, Faisal TT, et al. Ocular myiasis. J Ophthalmic Vis Res 2018;13(3):361-362. DOI: 10.4103/2008-322X.158891.

5. Wakamatsu TH, Pierre-Filho PT. Ophthalmomyiasis externa caused by Dermatobia hominis: a successful treatment with oral ivermectin. Eye (Lond) 2006;20(9):1088-1090. DOI: 10.1038/sj.eye.6702120.

6. Bayindir T, Cicek MT, Atambay M, et al. Cutaneous myiasis in a malignant wound of the head and neck region. J Craniofac Surg 2012;23(1):19-20. DOI: 10.1097/SCS.0b013e31824207d3.

7. Kokcam I, Saki CE. A case of cutaneous myiasis caused by Wohlfahrtia magnifica. J Dermatol 2005;32(6):459-463. DOI: 10.1111/j.13468138.2005.tb00780.x.

8. James MT. The flies that cause myiasis in man. U.S. Department of Agriculture Miscellaneous Publication No. 631. Washington, DC: USDA; 1947. pp. 1-175. DOI: 10.5962/bhl.title.65688.

9. Ruiz-Martinez I, Soler-Cruz MD, Benitez-Rodriguez R, et al. Postembryonic development of Wohlfahrtia magnifica (Schiner, 1862) (Diptera: Sarcophagidae). J Parasitol 1989;75(4):531-539.

10. Failoc-Rojas VE, Silva-Díaz H. Review of Cases and a patient report of myiasis with tracheostomy, Peru. Emerg Infect Dis 2016;22(3):563-565. DOI: 10.3201/eid2203.151631.

11. Mohajerin AH. Common cutaneous disorders of the eyelids. Cutis 1972;10:279.

12. Rietschel RL, Fowler JF, Fisher AA. Fisher's Contact Dermatitis, 5th ed. Philadelphia, PA: Lippincott Williams \& Wilkins; 2001.

13. Zug KA, Palay DA, Rock B. Dermatologic diagnosis and treatment of itchy red eyelids. Surv Ophthalmol 1996;40(4):293-306. DOI: 10.1016/ s0039-6257(96)82004-2. 